\title{
Two Faces of the Two-Phase Thermodynamic
}

\section{Model}

\author{
Ádám Madarász, ${ }^{* \dagger}$ Andrea Hamza, ${ }^{\dagger}$ Dávid Ferenc, ${ }^{\dagger, \ddagger}$ and Imre Bakó ${ }^{\dagger}$ \\ $\dagger$ †esearch Centre for Natural Sciences, Magyar Tudósok Körútja 2, H-1117 Budapest, \\ Hungary \\ $\ddagger$ Institute of Chemistry, ELTE, Eötvös Loránd University, Pázmány Péter sétány 1/A, \\ Budapest, H-1117, Hungary \\ E-mail: madarasz.adam@ttk.hu
}

\begin{abstract}
The quantum harmonic model and the two-phase thermodynamics method (2PT) are widely used to obtain quantum corrected properties such as isobaric heat capacities or molar entropies. 2PT heat capacities were calculated inconsistently in the literature. For water the classical heat capacity was also considered, but for organic liquids it was omitted. We reanalyzed the performance of different quantum corrections on the heat capacities of common organic solvents against experimental data. We have pointed out serious flaws in previous $2 \mathrm{PT}$ studies. The vibrational density of states was calculated incorrectly causing $39 \%$ relative error in diffusion coefficients and $45 \%$ error in the $2 \mathrm{PT}$ heat capacities. The wrong conversion of isobaric isochoric heat capacity also caused about $40 \%$ error but in the other direction. We have introduced the concept of anharmonic correction (AC) which is simply the deviation of the classical heat capacity from that of the harmonic oscillator model. This anharmonic contribution is around $+30-40 \mathrm{~J} / \mathrm{mol} / \mathrm{K}$ for water depending on the water model and $-8-10 \mathrm{~J} / \mathrm{mol} / \mathrm{K}$ for hydrocarbons and halocarbons. $\mathrm{AC}$ is unrealistically large, $+40 \mathrm{~J} / \mathrm{K} / \mathrm{mol}$ for alcohols
\end{abstract}


and amines indicating some deficiency of the OPLS force field. The accuracy of the computations was also assessed with the determination of the self-diffusion coefficients.

\section{Introduction}

Accounting for nuclear quantum effects is essential to obtain meaningful thermodynamic properties that are comparable to experimental observations. ${ }^{1}$ The most typical example is that zero point energies are indispensable in the determination of reaction free energies. The quantum harmonic oscillator model works quite well for small molecules and solid states, but the anharmonicity becomes significant in macromolecules, interfaces and liquids and the potential energy surfaces must be mapped using molecular dynamics or Monte Carlo simulation. Berens proposed to add quantum correction to the classically calculated properties using the harmonic oscillator model. ${ }^{2}$ Goddard improved this by the separation of different motions like translation rotations and vibrations and using different partition functions for each of them. ${ }^{3,4}$ This was abbreviated as two-phase thermodynamic (2PT) model referring to the gas phase and solid phase motions in contrast to the one-phase thermodynamic (1PT) method where only vibrations were considered. An anharmonic correction was also included in Berens' original idea, and thus we refer to that method as one-phase-thermodynamics with anharmonic correction $(1 \mathrm{PT}+\mathrm{AC})$.

2PT and 1PT + AC methods were successfully applied for the calculation of thermody-

namic properties of several systems such as Lennard-Jones fluids, ${ }^{3,5}$ water, ${ }^{2,4,6-15}$ aqueous solutions, ${ }^{16,17}$ molten salts, ${ }^{18}$ organic liquids,${ }^{19-21}$ carbon dioxide, ${ }^{22}$ urea,${ }^{23}$ ionic liquids, ${ }^{24-27}$ carbohydrates, ${ }^{28}$ cellulose, ${ }^{29}$ mixtures ${ }^{30}$ and interfaces. ${ }^{31-36}$ Lately, $2 \mathrm{PT}$ was used for the definition of the Frenkel line. ${ }^{37-40}$ Both 1PT/2PT methods are still in continuous development in respect of accuracy and applicability. ${ }^{41-49}$

The 2PT method is the most excellent in the calculation of absolute entropy even from short trajectories. Although the heat capacity is strictly determined from the temperature 
dependence of entropy according to the laws of thermodynamics, the calculation of the $2 \mathrm{PT}$ heat capacity is not as consistent in the literature as the computation of the 2PT entropy. The 2PT abbreviation refers to two conceptually different calculation procedures of the heat capacity in different articles. The classical heat capacities were also taken into account in the calculation of the $2 \mathrm{PT}$ heat capacity of water, $9,10,17,33$ but in the case of organic solvents classical heat capacities were discarded. ${ }^{19-21}$ According to refs 19-21 we refer to 1PT and 2PT heat capacities that do not contain anharmonic corrections calculated from classical values. In previous studies there was no systematic comparison of the effect of this anharmonic correction. In the present study we fill this gap and analyse the $2 \mathrm{PT}$ and $1 \mathrm{PT}+\mathrm{AC}$ methods in more details.

Here we focus on heat capacities to evaluate different types of quantum corrections because it contains large nuclear quantum effect and there are accurate experimental data that can be used for the benchmark of force fields. ${ }^{6,17,19-21,25-27,29,50-58}$ In contrast to enthalpy or Gibbs energy, heat capacity is an absolute quantity meaning that there is no need to set the zero point. Additionally, the isobaric heat capacity is a state function, so if we know the $c_{p}$ as a function of $T$ and $p$, the other state functions such as the enthalpy and entropy can be calculated as well. Previously, quantum corrected thermodynamic properties of organic solvents were investigated in two systematic studies by Pascal, and Caleman. ${ }^{19,20}$ For the same solvents they found similar results: the 2PT heat capacities were in good agreements with the experimental data. Both studies showed that OPLS force field gave better results than other general force fields such as GAFF or CHARMM. We reanalyzed 113 organic solvents from ref 20 to test further the $1 \mathrm{PT}+\mathrm{AC}$ and $2 \mathrm{PT}$ methods. 21 solvents were omitted from the analysis of the heat capacities, because their calculated self-diffusion coefficients were under $10^{-10} \mathrm{~m}^{2} / \mathrm{s}$ indicating that these systems do not behave like a real fluid but an amorphous solid. 


\section{Theory}

For the determination of the quantum corrected thermodynamic properties the velocity autocorrelation functions (VACF) are computed from molecular dynamics simulations that can be defined as follows:

$$
\operatorname{VACF}(t)=\frac{\int_{0}^{\infty} m v(t+\tau) \cdot v(\tau) \mathrm{d} \tau}{\int_{0}^{\infty} m v(\tau) \cdot v(\tau) \mathrm{d} \tau}
$$

where $m$ is the atomic mass and $v$ is the velocity as a function of time $(t)$. With this definition the autocorrelation function is always 1 at zero time i.e. $\operatorname{VACF}(0)=1$. The vibrational density of states (VDOS) is the Fourier transform of the autocorrelation function (VACF)

$$
\operatorname{VDOS}(\nu)=\mathcal{F}_{t}\{\operatorname{VACF}(t)\}(\nu)=2 \int_{0}^{+\infty} \operatorname{VACF}(t) \cdot \cos (2 \pi \nu t) \mathrm{d} t
$$

where $\nu$ is the frequency.

The Fourier transform of VDOS equals to the VACF:

$$
\operatorname{VACF}(t)=\mathcal{F}_{\nu}\{\operatorname{VDOS}(\nu)\}(t)=2 \int_{0}^{+\infty} \operatorname{VDOS}(\nu) \cdot \cos (2 \pi \nu t) \mathrm{d} \nu
$$

If we set $t=0$ in eq 3 then we obtain the norm of VDOS:

$$
2 \int_{0}^{\infty} \operatorname{VDOS}(\nu) \mathrm{d} \nu=\int_{-\infty}^{\infty} \operatorname{VDOS}(\nu) \mathrm{d} \nu=\mathcal{F}_{\nu}\{\operatorname{VDOS}(\nu)\}(0)=\operatorname{VACF}(0)=1
$$

Originally Berens proposed that the quantum corrected density of states can be determined by the multiplication of VDOS with an appropriate weight function $w:^{2}$

$$
\operatorname{VDOS}^{\mathrm{q}}(\nu)=\operatorname{VDOS}(\nu) \cdot w(\nu)
$$


In the $1 \mathrm{PT}$ method there is no separation of motions, all are considered as vibrations. The quantum weight function for the heat capacity is 59

$$
w_{\mathrm{vib}}^{c_{V}}(\nu)=\exp (\beta h \nu)\left(\frac{\beta h \nu}{1-\exp (\beta h \nu)}\right)^{2}
$$

where $\beta=\left(k_{\mathrm{B}} T\right)^{-1}, k_{\mathrm{B}}$ is the Boltzmann constant, $T$ is the temperature, $h$ is the Planck constant. Thus the isochoric heat capacity can be calculated as

$$
c_{V}^{1 \mathrm{PT}}=2 f R \int_{0}^{\infty} \operatorname{VDOS}(\nu) \cdot w_{\mathrm{vib}}^{c_{V}}(\nu) \mathrm{d} \nu,
$$

where $R$ is the universal gas constant, and $f=3 N$ is the number of degrees of freedom of an $N$-atomic molecule.

Gaseous motions are separated from vibrations in the $2 \mathrm{PT}$ method. The total VDOS is decomposed into two terms, solid and gaseous components:

$$
\operatorname{VDOS}(\nu)=\operatorname{VDOS}_{\mathrm{sol}}(\nu)+\operatorname{VDOS}_{\mathrm{gas}}(\nu)
$$

The gaseous component is determined by $\operatorname{VDOS}(0)$ and the fluidicity factor $f_{\mathrm{m}}$ :

$$
\operatorname{VDOS}_{\text {gas }}(\nu)=\frac{\operatorname{VDOS}(0)}{1+\left(\frac{\pi N \operatorname{VDOS}(0)}{2 f_{\mathrm{m}} N_{\mathrm{mol}}} \nu\right)^{2}}
$$

where $N_{\text {mol }}$ is the number of molecules. With the definition of eqs 1 and 2, we obtain molar quantities and $N_{\mathrm{mol}}=1$. In an improved version of $2 \mathrm{PT}$ the gaseous and solid components of the VDOS are determined for both translation and rotation. ${ }^{4}$ This decomposition, however, is not really needed because the gaseous component of the rotation is zero as in the case of vibration. See the derivation in the Supporting Information.

Different weight functions are used for the different motions in the calculation of $2 \mathrm{PT}$ heat capacity: ${ }^{19,20}$ 


$$
c_{V}^{2 \mathrm{PT}}=2 f R \int_{0}^{\infty}\left[\operatorname{VDOS}_{\mathrm{sol}}(\nu) w_{\mathrm{vib}}^{c_{V}}(\nu)+\operatorname{VDOS}_{\text {gas }} w_{\text {gas }}^{c_{V}}(\nu)\right] \mathrm{d} \nu
$$

The weight function of the gaseous component is $1 / 2$ for the heat capacity.

In the $1 \mathrm{PT}+\mathrm{AC}$ method a quantum correction $\left(c_{V}^{\Delta}\right)$ is added to the classical isochoric heat capacity $\left(c_{V}^{\mathrm{cl}}\right)^{2}$ as Berens et al. proposed originally: ${ }^{2}$

$$
c_{V}^{1 \mathrm{PT}+\mathrm{AC}}=c_{V}^{\mathrm{cl}}+c_{V}^{\Delta}
$$

The quantum correction can be determined from the quantum harmonic weight function. $c_{V}^{\Delta}$ given by

$$
c_{V}^{\Delta}=2 f R \int_{0}^{\infty} \operatorname{VDOS}(\nu) \cdot\left(w^{c_{V}}(\nu)-1\right) \mathrm{d} \nu
$$

If the integral terms are partitioned differently then the 1PT $+\mathrm{AC}$ notation becomes apparent:

$$
\begin{aligned}
c_{V}^{1 \mathrm{PT}+\mathrm{AC}} & =2 f R \int_{0}^{\infty} \operatorname{VDOS}(\nu) \cdot w^{c_{V}}(\nu) \mathrm{d} \nu+c_{V}^{\mathrm{cl}} \\
& -2 f R \int_{0}^{\infty} \operatorname{VDOS}(\nu) \mathrm{d} \nu=c_{V}^{1 \mathrm{PT}}+c_{V}^{\mathrm{AC}}
\end{aligned}
$$

where the second term is the anharmonic correction:

$$
c_{V}^{\mathrm{AC}}=c_{V}^{\mathrm{cl}}-2 f R \int_{0}^{\infty} \operatorname{VDOS}(\nu) \mathrm{d} \nu=c_{V}^{\mathrm{cl}}-f R
$$

The 1PT $+\mathrm{AC}$ heat capacity is actually a sum of three terms: the heat capacity of $f$ classical harmonic oscillators plus an anharmonic- and a quantum correction:

$$
c_{V}^{1 \mathrm{PT}+\mathrm{AC}}=c_{V}^{\mathrm{cl}}+c_{V}^{\Delta}=c_{V}^{1 \mathrm{PT}}+c^{\mathrm{AC}}=f R+c^{\mathrm{AC}}+c_{V}^{\Delta}
$$

Jorgensen proposed to correct the classical heat capacity by the estimation of the intramolecular component using the ideal gas value taken from experiments or ab initio cal- 
culations. ${ }^{50,51}$ If a given force field reproduces the experimental heat capacity of the gas accurately then Jorgensen's approach should give a similar value to the 1PT+AC method. Some deviation may occur if the frequencies of the intramolecular vibrations differ in the liquid and gas phases.

Recently we have shown that Berens' original idea about the quantum correction on thermodynamic properties can be extended to structural properties if the quantum correction is applied in time domain instead of frequency domain. ${ }^{60,61}$ Our technique, the generalized smoothed trajectory analysis (GSTA) gives identical results for thermodynamics properties as $1 \mathrm{PT}+\mathrm{AC}$. For instance the heat capacity can be obtained from the VACF directly:

$$
c_{V}^{1 \mathrm{PT}+\mathrm{AC}}=2 f R \int_{0}^{+\infty} \gamma^{c_{V}}(\tau) \cdot \operatorname{VACF}(\tau) \mathrm{d} \tau+c_{V}^{\mathrm{AC}}
$$

where $\gamma^{c_{V}}$ is the Fourier transform of the weight function in eq 6 :

$$
\gamma^{c_{V}}(\tau)=\frac{2 \pi^{2}}{\beta h} \operatorname{csch}^{2}\left(\frac{2 \pi^{2}}{\beta h} \tau\right)\left(\frac{2 \pi^{2}}{\beta h} \tau \operatorname{coth}\left(\frac{2 \pi^{2}}{\beta h} \tau\right)-1\right)
$$

where csch is the hyperbolic cosecant function. This formalism allows a much more effective calculation, because there is no need to calculate the VDOS.

The isobaric heat capacity can be determined from the isochoric heat capacity by employing the relation

$$
c_{p}=c_{V}+\frac{T M \alpha_{p}^{2}}{\rho \kappa_{T}}
$$

where $\alpha_{p}$ denotes the thermal expansion coefficient, $M$ is the relative molar mass, $\rho$ is the density and $\kappa_{T}$ is the isothermal compressibility. The isobaric $1 \mathrm{PT}+\mathrm{AC}$ heat capacity is computed as a sum of the classical isobaric heat capacity and the quantum correction from eq 15 and the latter can be determined from VACF or VDOS according to eqs 7 and 16:

$$
c_{p}^{1 \mathrm{PT}+\mathrm{AC}}=c_{p}^{\mathrm{cl}}+c_{V}^{\Delta}
$$


2PT can also be combined with an anharmonic correction that satisfies the correspondence principle:

$$
c_{V}^{2 \mathrm{PT}+\mathrm{AC}}=c_{V}^{2 \mathrm{PT}}+c_{V}^{\mathrm{cl}}-3 R\left(N-N_{\mathrm{mol}} f_{\mathrm{m}} / 2\right)
$$

This definition of $2 \mathrm{PT}+\mathrm{AC}$ heat capacity may correspond to previous $2 \mathrm{PT}$ calculations in the literature where the classical heat capacities were also taken into account. ${ }^{9,10,17,22,33}$

\section{Methods}

We performed $10.6 \mathrm{~ns}$ long $N p T$ simulations to determine the isobaric heat capacities and self-diffusion coefficients by using the GROMACS simulation software. ${ }^{62}$ The settings and inputs were taken from ref 20. (The input files can be found in the Supporting Information). The cubic box always contained 1000 molecules. $1.1 \mathrm{~nm}$ cutoff was employed for the intermolecular interactions. The particle mesh Ewald algorithm was used for the computation of the Coulomb interactions. The time constants of the Nose-Hoover thermostat and the Parrinello-Rahman barostat were 1.0 and 5.0 ps, respectively. To determine the classical heat capacity including all vibrations, no constraints were applied on bonds, they remained flexible, and thus 0.2 fs time step was used. 2PT heat capacities were calculated with the "dos" analysis tool of GROMACS. The classical heat capacity was determined from the fluctuation of enthalpy

$$
c_{p}^{\mathrm{cl}}=\left(\frac{\left\langle\partial H^{2}\right\rangle}{R T^{2}}\right)_{p}
$$

\section{Results and Discussion}

\subsection{Heat Capacity}

According to the correspondence principle the quantum calculations should agree with the classical results as the $h$ Planck constant formally approaches zero. The 1PT model gives 
$f R$ for the heat capacity in the classical limit. Applying the classical weight functions of 1 and $1 / 2$ in eq 10 it is easy to see, that the $2 \mathrm{PT}$ model can give values between $f R / 2$ and $f R$ for the isochoric heat capacities in the classical limit. The $1 \mathrm{PT}+\mathrm{AC}$ and $2 \mathrm{PT}+\mathrm{AC}$ models always satisfy the correspondence principle in contrast with the 1PT or 2PT methods:

$$
\lim _{h \rightarrow 0} c_{p}^{1 \mathrm{PT}+\mathrm{AC}}=\lim _{h \rightarrow 0} c_{p}^{2 \mathrm{PT}+\mathrm{AC}}=c_{p}^{\mathrm{cl}}
$$

This also implies that the technique is able to describe the effects of anharmonic motions. The 1PT and 2PT isochoric heat capacities for a rigid water model with 3 translational and 3 rotational degrees of freedom cannot be higher than $6 R=49.9 \mathrm{~J} / \mathrm{mol} / \mathrm{K}$. The fact that in ref $9,10,17,33$ the calculated heat capacities are in the range of 57 and $81 \mathrm{~J} / \mathrm{K} / \mathrm{mol}$ which is significantly larger than the theoretical limit of $49.9 \mathrm{~J} / \mathrm{K} / \mathrm{mol}$ implies that anharmonic contribution was also considered. Since the experimental isochoric heat capacity is 74.5 $\mathrm{J} / \mathrm{K} / \mathrm{mol}$, the anharmonic contribution is at least $24.6 \mathrm{~J} / \mathrm{K} / \mathrm{mol} .{ }^{63}$ From previous simulations the calculated anharmonic correction is around 30-40 J/K/mol depending on the water model. ${ }^{60}$

In our previous study we showed that the $1 \mathrm{PT}+\mathrm{AC}$ heat capacity can be significantly overestimated if the left Riemann sum is used instead of the trapezoidal rule in the computation of VDOS in eq 2. ${ }^{60}$ To check whether this numerical error can occur in the calculation of the 2PT heat capacities, we tested thoroughly the "dos" analysis tool of the GROMACS that was used in ref 20. The default Fast Fourier Transformation routine in GROMACS applies the left Riemann rule but we also implemented a simple trapezoidal integral rule. We analyzed the effect of the different algorithms on methanol. Decreasing the time interval of the integration, the trapezoidal integral converged rapidly at $5 \mathrm{fs}$, meanwhile the default left Riemann sum gave the correct $2 \mathrm{PT}$ heat capacity, $51.6 \mathrm{~J} / \mathrm{K} / \mathrm{mol}$ only in the $\Delta t \rightarrow 0$ limit (see Figure 1). At 4 fs time interval, which is generally used in 2PT calculations, the heat capacity is $73.4 \mathrm{~J} / \mathrm{K} / \mathrm{mol}$ which means $40 \%$ overestimation of the correct value. This agrees 
well with the result of $75.8 \mathrm{~J} / \mathrm{K} / \mathrm{mol}$ from ref 20 . There is a breaking point for the $2 \mathrm{PT}$ heat capacities at $9 \mathrm{fs}$. This is due to the fact that the period of $\mathrm{OH}$ vibration is exactly $9.0 \mathrm{fs}$, and this coincidence causes large uncertainty in the calculation of VDOS at zero frequency. The convergence of the 1PT method is also shown with the trapezoidal formula, and at $4 \mathrm{fs}$ the $1 \mathrm{PT}$ heat capacity is also converged with $60.7 \mathrm{~J} / \mathrm{K} / \mathrm{mol}$.

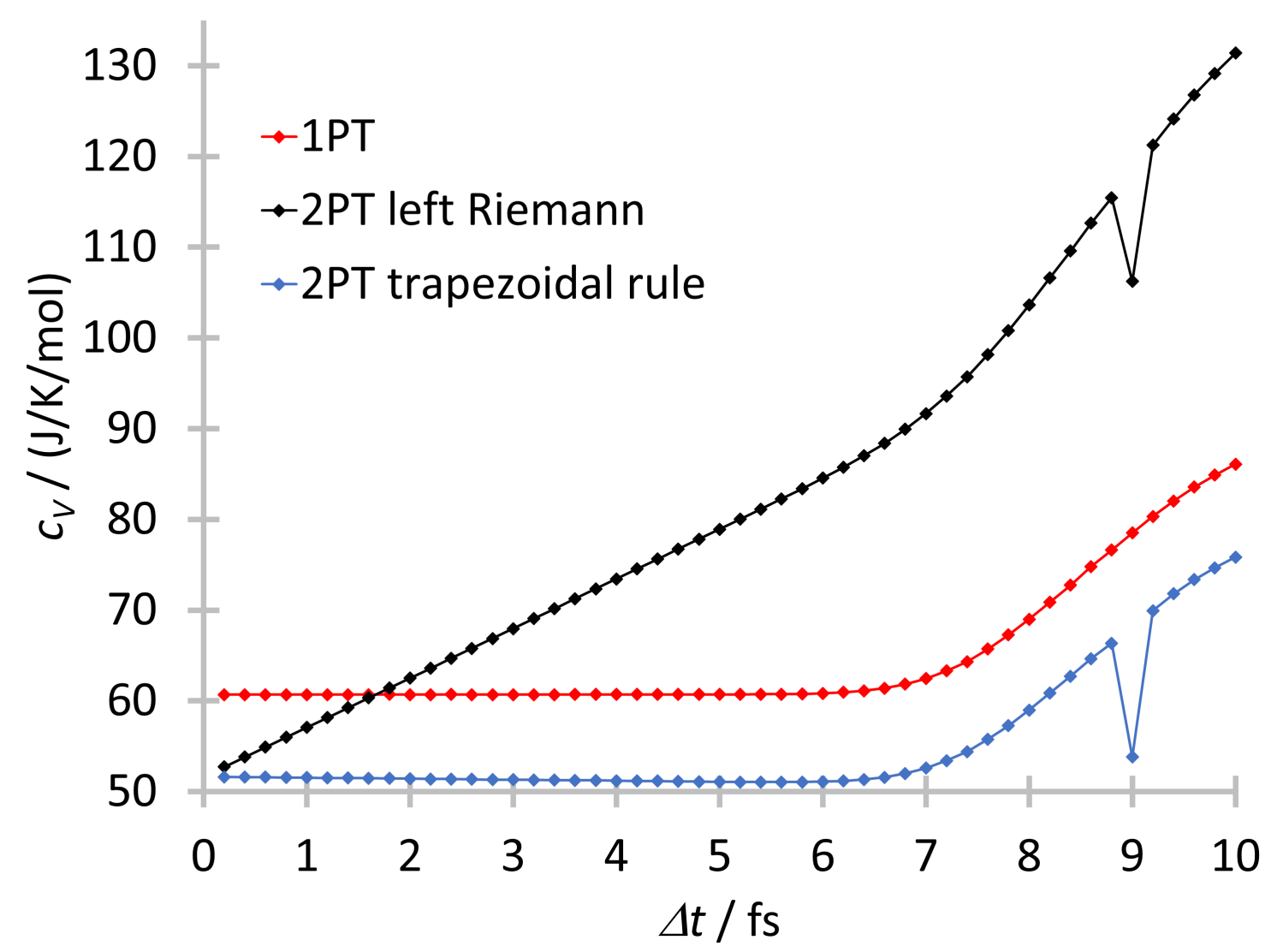

Figure 1: Convergence of the isochoric heat capacity of methanol as a function of time interval

When we recalculated the heat capacities of 9 common solvents from ref 19 and 20 with both integral formulas, we reproduced the literature data but we obtained $45 \%$ lower heat capacities with the correct integral formula (see the Supporting Information). In ref 19 and 20 the 2PT heat capacities are similar to each other for the same solvents with OPLS force field, which implies that in both works the same (incorrect) integration routine was used.

Surprisingly, in these previous works excellent correlations were found between the $2 \mathrm{PT}$ 
and experimental isobaric heat capacities. How is it possible that such a good correlation has been achieved, if the values were overestimated by $45 \%$ ? It seems that there was an (un)fortunate error cancellation, where the opposite error is connected to the conversion between the isobaric and isochoric heat capacities in eq 18 .

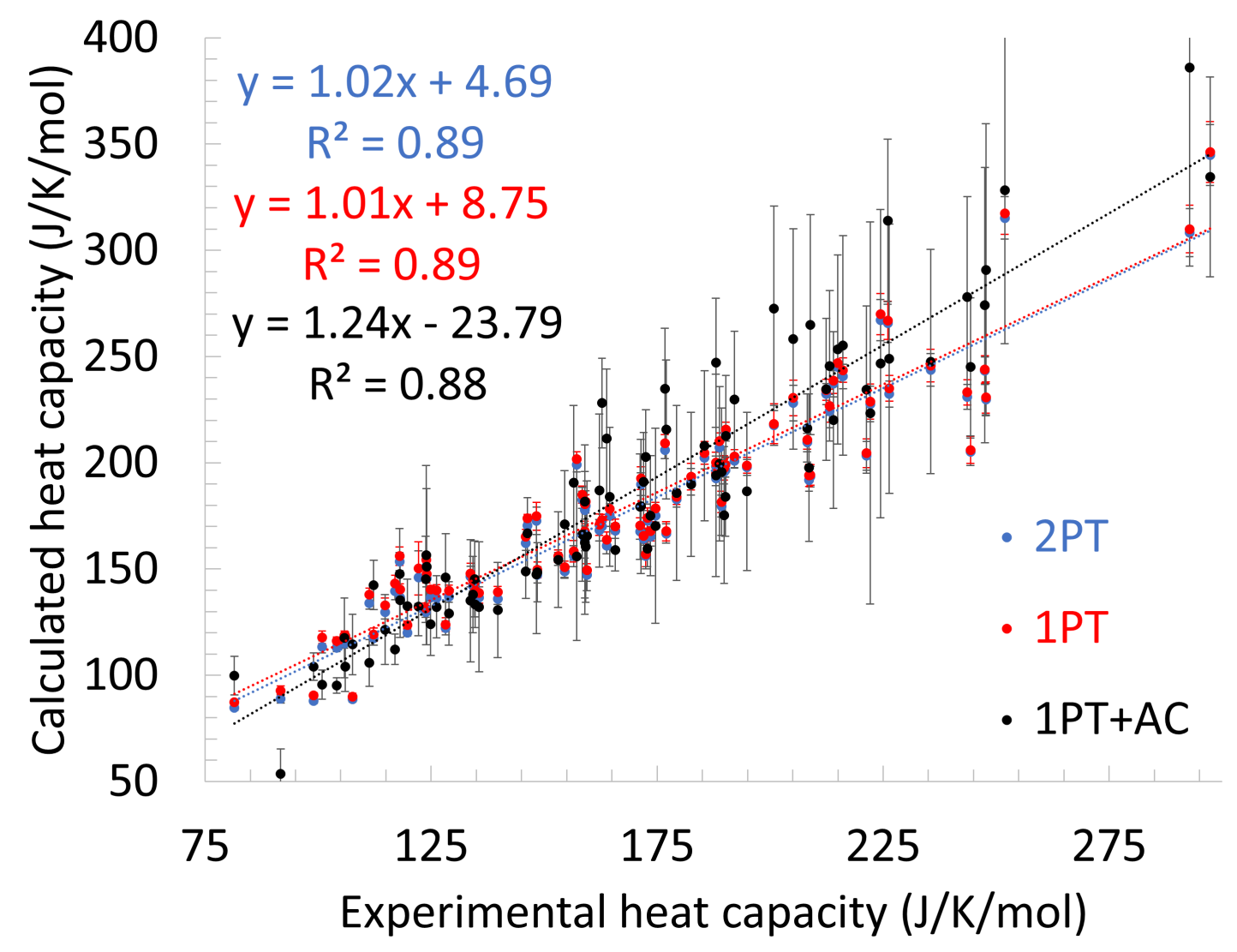

Figure 2: Calculated vs. experimental isobaric heat capacities

In ref 20 the $c_{p}-c_{V}$ difference is always smaller than $0.1 \mathrm{~J} / \mathrm{K} / \mathrm{mol}$, and in ref 19 this correction was not larger than $1.2 \mathrm{~J} / \mathrm{K} / \mathrm{mol}$. We recalculated the $c_{p}-c_{V}$ differences for the organic liquids from ref 20 and we obtained orders of magnitude higher values. In our computations the average difference is $38.4 \mathrm{~J} / \mathrm{K} / \mathrm{mol}$, and the heat capacity ratio is 1.31 . For a few molecules there are direct experimental data for the isochoric heat capacities (see the Supporting Information). For instance the $c_{p}-c_{V}$ differences of methanol and ethanol are 14 and $11 \mathrm{~J} / \mathrm{K} / \mathrm{mol}$, respectively. ${ }^{64,65}$ This supports that we calculated correctly the $c_{p}-c_{V}$ values. 
We also tried to reproduce the heat capacity of methanol from ref 19 using the same simulation software, LAMMPS and the same program code that determined the heat capacities. Although we obtained the same total VDOS with LAMMPS as we did with GROMACS, the solid part was quite different (see the Supporting Information). It turned out that the solid part of the VDOS function is calculated incorrectly in GROMACS, because the number of atoms was used instead of the number of molecules in eq 8. In Pascal's current 2PT code the FFT algorithms give the numerically exact results and the classical heat capacity is also taken into account in the final heat capacity.

We also determined the $2 \mathrm{PT}+\mathrm{AC}$ heat capacities according to eq 20 . The $2 \mathrm{PT}+\mathrm{AC}$ heat capacities are always slightly higher than the $1 \mathrm{PT}+\mathrm{AC}$ heat capacities, and the maximum difference is $0.33 \mathrm{~J} / \mathrm{K} / \mathrm{mol}$ which is definitely smaller than the uncertainty of the calculations. Since the $2 \mathrm{PT}+\mathrm{AC}$ heat capacities are almost identical with the $1 \mathrm{PT}+\mathrm{AC}$ heat capacities (see the Supporting Information), we discuss only the $1 \mathrm{PT}+\mathrm{AC}$ heat capacities in the rest of the paper.

The correctly calculated isobaric heat capacities are shown in Figure 2 as a function of experimental values. The overall correlations are good for the predicted and experimental heat capacities, the $R^{2}$ is about 0.9 for all three methods. From the fitted lines it can be seen that the slope of the 1PT and 2PT are almost perfect 1.01 and 1.02 respectively, but for $1 \mathrm{PT}+\mathrm{AC}$ the slope is 1.24 . Both $1 \mathrm{PT}$ and $2 \mathrm{PT}$ overestimates the isobaric heat capacities. The 2PT model yields systematically lower heat capacities than 1PT with around $2.1 \mathrm{~J} / \mathrm{K} / \mathrm{mol}$, which can be easily explained by the fact the $2 \mathrm{PT}$ considers gaseous motions as well that have smaller heat capacity than vibrations ( $R / 2$ vs. $R$ per degree of freedom). The methods perform differently for different types of compounds, and even their relative goodness is varying.

The mean absolute deviations for different types of molecules are shown in Figure 3. For all the compounds the error is $20 \mathrm{~J} / \mathrm{K} / \mathrm{mol}$ for the $1 \mathrm{PT}+\mathrm{AC}$ method. The error is smaller with 1PT and even smaller with the 2PT method. For hydrocarbons, organosulfures, 
halocarbons and heteroaromatics the $1 \mathrm{PT}$ performs the worst, and the $1 \mathrm{PT}+\mathrm{AC}$ and $2 \mathrm{PT}$ performs similarly better. For amines, ethers, alcohols and ketones the 1PT $+\mathrm{AC}$ performs the worst, and the $1 \mathrm{PT}$ and $2 \mathrm{PT}$ methods perform much better. The large errors of the $1 \mathrm{PT}+\mathrm{AC}$ heat capacities may originate from the deficiency of the force field and/or from the inaccuracy of the 1PT $+\mathrm{AC}$ method. To separate these two errors, we investigated the classical limits which characterizes the failure of the method.

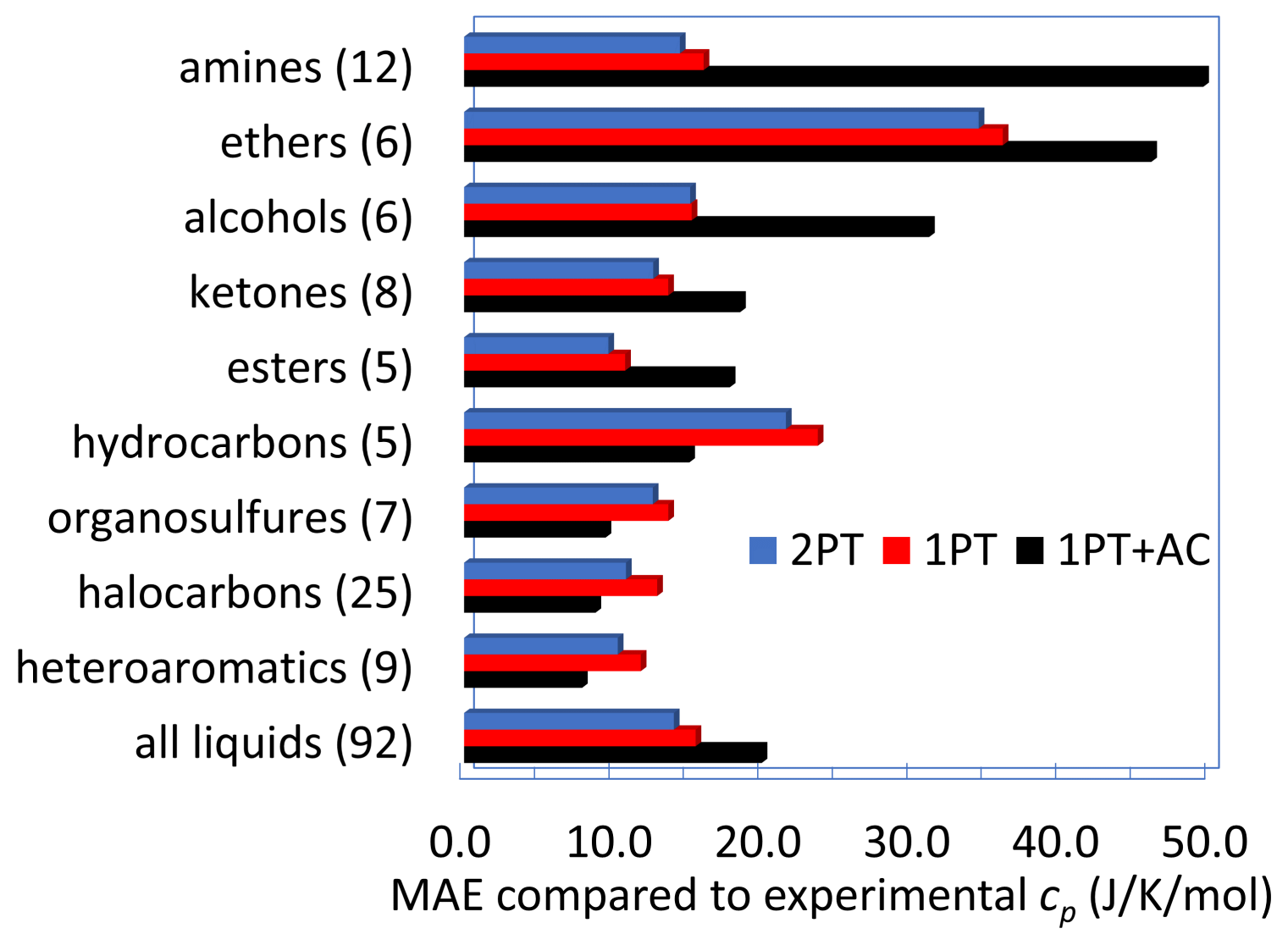

Figure 3: Mean absolute errors (MAE) of the calculated isobaric heat capacities compared to experimental data.

As mentioned above the 1PT and 2PT models do not satisfy the correspondence principle, they cannot reproduce the classical heat capacities of anharmonic cases. To quantify these deviations the mean error of the reproduction of the classical heat capacities is shown for the $1 \mathrm{PT}$ and $2 \mathrm{PT}$ methods in Figure $4.1 \mathrm{PT}+\mathrm{AC}$ and $2 \mathrm{PT}+\mathrm{AC}$ are not shown because their errors are zero according to eq 22. This kind of error of $1 \mathrm{PT}$ equals the negative of 
the anharmonic correction 1PT + AC model. 2PT always underestimates the classical heat capacity with $8.2 \mathrm{~J} / \mathrm{mol} / \mathrm{K}$ in average which is comparable to the mean absolute error relative to the experiments, $14.2 \mathrm{~J} / \mathrm{mol} / \mathrm{K}$. The $1 \mathrm{PT}$ method overestimates the classical heat capacity for the heteroaromatics, halocarbons, organosulfures and hydrocarbons and underestimates for the other compound groups. This classification correlates perfectly with the relative performance of $1 \mathrm{PT}$ and $1 \mathrm{PT}+\mathrm{AC}$ in Figure 3 . If the $1 \mathrm{PT}$ heat capacity agrees better with the experiment than the $1 \mathrm{PT}+\mathrm{AC}$, then it means that the anharmonicity is described incorrectly by the force field. The Lennard-Jones potential is known to be too repulsive and this may cause inaccuracies when stronger attractive interactions are also present. The uncertainty of the anharmonicity is too large with the OPLS force field for the aliphatic N and $\mathrm{O}$ compounds and this is why the $2 \mathrm{PT}$ method estimates the heat capacity of organic liquids more accurately. These results suggest that the effect of anharmonicity is significantly smaller than the quantum effect on the heat capacity of the organic liquids. In the $1 \mathrm{PT}+\mathrm{AC}$ method this means that the magnitude of the anharmonic correction is smaller than that of the quantum correction in eq 15. 


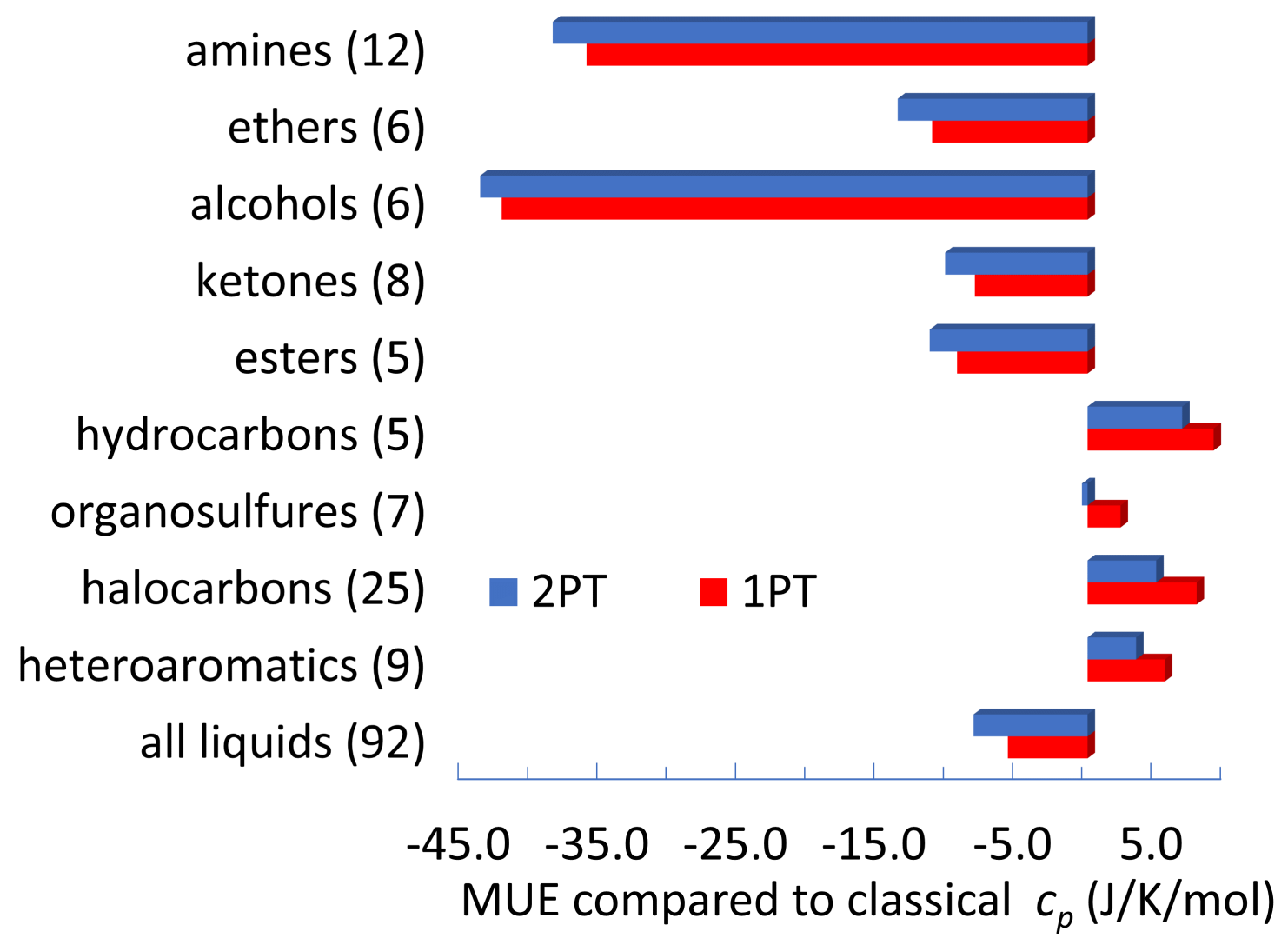

Figure 4: Mean unsigned errors (MUE) of the isobaric heat capacities compared to the classical limit.

Caleman and Pascal concluded from their studies that the reproduction of the experimental heat capacities could be improved by better description of the force constants of bonds and angles. ${ }^{19,20}$ This is true for the quantum correction, but the anharmonic correction can be adjusted with the non-bonding parameters. Our results indicate that the thermodynamic properties are more sensitive to the intermolecular interactions than to the intramolecular interactions. This is in line with the general experiences that in the simulation of the organic liquids the bond lengths and angles can be constrained at room temperature.

\subsection{Self-Diffusion}

To estimate the consistency of the calculations of VACF and VDOS functions we computed the self-diffusion coefficients with two different methods. ${ }^{66}$ First, we determined $D_{s}$ from the 
VACF:

$$
D_{\mathrm{s}}=\frac{\left\langle v^{2}\right\rangle}{3} \lim _{t \rightarrow \infty} \int_{0}^{t} \operatorname{VACF}(\tau) \mathrm{d} \tau
$$

The self-diffusion coefficient can also be calculated from the mean square displacement of the atoms using the Einstein equation:

$$
D_{\mathrm{s}}=\lim _{t \rightarrow \infty} \frac{\partial\left\langle(x(t)-x(0))^{2}\right\rangle}{6 \partial t}
$$

We computed the self-diffusion coefficients according to these equations with a time lag of 10 ps. The two complementary approaches gave almost identical results (MAE = $\left.0.05 \cdot 10^{-9} \mathrm{~m}^{2} \mathrm{~s}^{-1}, R^{2}=0.998\right)$ which validates how we calculated the VACF and VDOS functions (see the Supplementary Information). If we use the less accurate left Riemann sum in eq 23 with the time interval of 4 fs then the self-diffusion coefficients are overestimated by $39 \%$. The self-diffusion coefficients were also determined from the 10 ns simulations using eq 24. Comparing to the available experimental data the mean absolute error is $0.62 \cdot 10^{-9} \mathrm{~m}^{2} \mathrm{~s}^{-1}$ for 31 liquids, which means significant correlation $\left(R^{2}=0.79\right)$ (see Figure 5$)$. In most cases the differences between the 10 ps and 10 ns values are larger than the uncertainty of the self-diffusion coefficients. Previous studies already showed that 10 ps time lag is not long enough for converged self-diffusion coefficients (see in ref 67 and further references therein.) The application of $10 \mathrm{~ns}$ time lag instead of $10 \mathrm{ps,} \mathrm{however,} \mathrm{do} \mathrm{not} \mathrm{improve} \mathrm{the} \mathrm{accuracy}$ of the self-diffusion coefficient compared to the experiment. Actually, the mean absolute error is slightly lower, $0.55 \cdot 10^{-9} \mathrm{~m}^{2} \mathrm{~s}^{-1}$ using $10 \mathrm{ps}$ long VACF functions, and the correlation coefficient is almost the same $\left(R^{2}=0.77\right)$.

\subsection{Efficiency of Different Algorithms}

Computation of the heat capacity from a converged VDOS function is a memory-intensive calculation since all of the velocities need to be read from several picosecond long trajec- 
tories with time intervals of a few femtoseconds. This computational cost can be reduced significantly with the GSTA approach. In the present case, if the VACF function is corrected with eq 16, it is enough to compute the VACF 68 fs long, instead of the 10 ps length used for the determination of the VDOS.

The calculation of the self-diffusion coefficient from a trajectory is orders of magnitude more expensive with the Green-Kubo method than with the Eisntein equation. While in the first case we needed to compute $10 \mathrm{ps}$ long VACF with a resolution of $4 \mathrm{fs}$, in the latter case it was enough to calculate the mean squared displacement at 8 and 12 ps. Moreover, the calculation of VACF is almost unfeasible with a time lag of 100 ps or longer, when the self-diffusion coefficient converges.

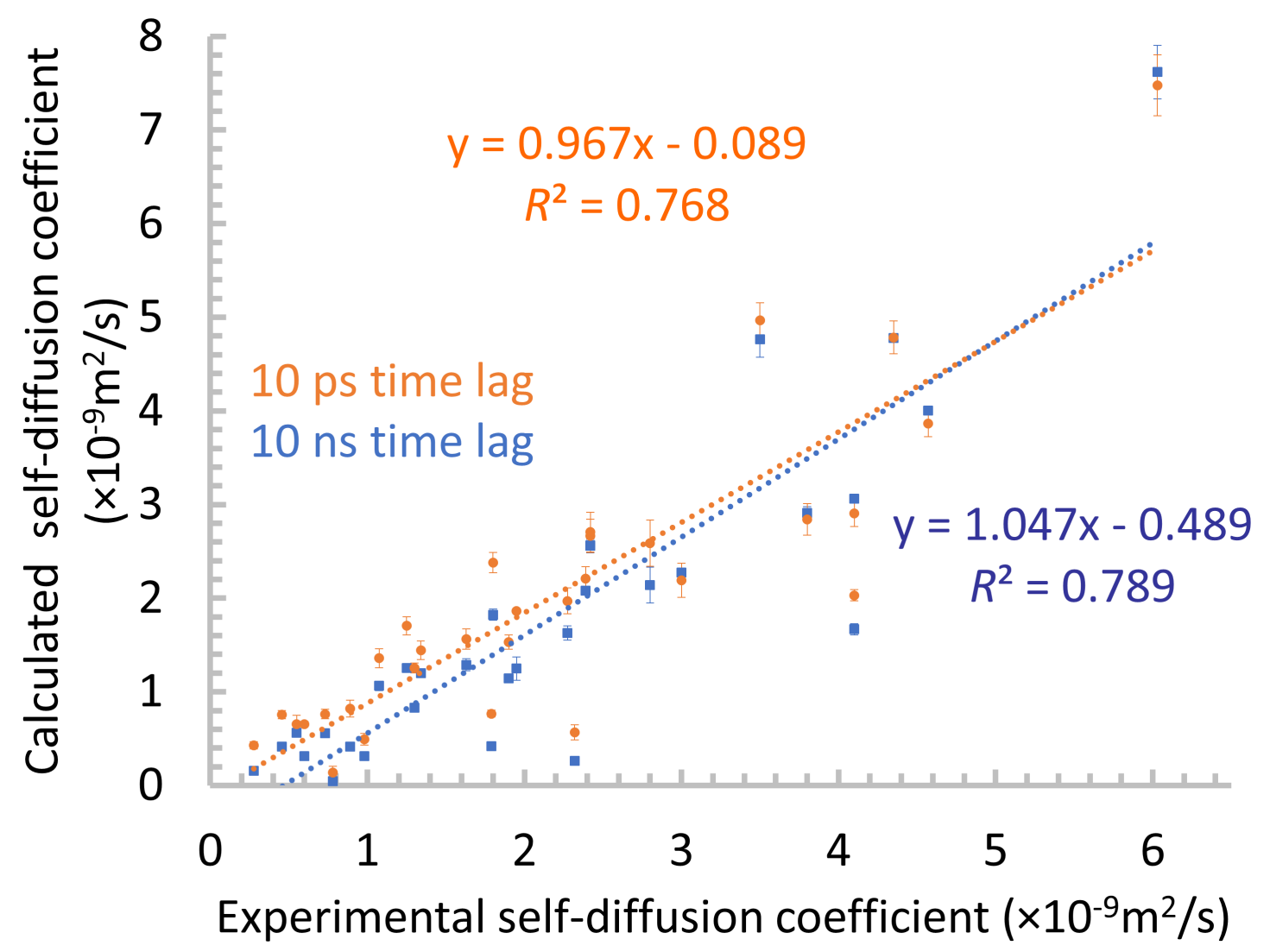

Figure 5: Calculated vs. experimental self-diffusion coefficients for 31 organic solvents 


\section{Conclusions}

As a summary, we compared the $2 \mathrm{PT}$ and $1 \mathrm{PT}+\mathrm{AC}$ heat capacities. We pointed out that previous $2 \mathrm{PT}$ heat capacities in the literature were calculated incorrectly. ${ }^{19-21}$ We think that the correct $2 \mathrm{PT}$ heat capacities include anharmonic correction (denoted $2 \mathrm{PT}+\mathrm{AC}$ in the

present paper). The right program code is given in the Supporting Information to calculate the correct $2 \mathrm{PT}, 1 \mathrm{PT}$ and $1 \mathrm{PT}+\mathrm{AC}$ methods by using the GROMACS software. Based on our benchmark calculations we suggest to use different methods for different purposes. Despite the 2PT method does not satisfies the correspondence principle, it can give reasonable estimation for thermodynamic properties of organic liquids. If someone wants to benchmark force fields, or develop new force field parameters it is recommended to use the $1 \mathrm{PT}+\mathrm{AC}$ method which accounts the anharmonicity correctly. Our results help to improve the accuracy of the calculated thermodynamic properties of large systems, and with the use of more efficient algorithms even larger systems can be investigated.

\section{Acknowledgement}

This work was supported by the National Research, Development and Innovation Office of Hungary (NKFI, Grant No. K-124885 and PD-121070). A.M. acknowledges the János Bolyai Scholarship from Hungarian Academy of Sciences.

\section{Supporting Information Available}

The details of the simulations along with the program codes, input files and results are available in the Supporting Information. 


\section{References}

(1) Collinge, G.; Yuk, S. F.; Nguyen, M.-T.; Lee, M.-S.; Glezakou, V.-A.; Rousseau, R. Effect of Collective Dynamics and Anharmonicity on Entropy in Heterogenous Catalysis: Building the Case for Advanced Molecular Simulations. ACS Catal. 2020, 10, 9236-9260.

(2) Berens, P. H.; Mackay, D. H. J.; White, G. M.; Wilson, K. R. Thermodynamics and quantum corrections from molecular dynamics for liquid water. J. Chem. Phys. 1983, 79, 2375-2389.

(3) Lin, S.-T.; Blanco, M.; Goddard, W. A. The two-phase model for calculating thermodynamic properties of liquids from molecular dynamics: Validation for the phase diagram of Lennard-Jones fluids. J. Chem. Phys. 2003, 119, 11792-11805.

(4) Lin, S.-T.; Maiti, P. K.; Goddard, W. A. Two-Phase Thermodynamic Model for Efficient and Accurate Absolute Entropy of Water from Molecular Dynamics Simulations. J. Phys. Chem. B 2010, 114, 8191-8198.

(5) Chari, S. S. N.; Dasgupta, C.; Maiti, P. K. Scalar activity induced phase separation and liquid-solid transition in a Lennard-Jones system. Soft Matter 2019, 15, 7275-7285.

(6) Laury, M. L.; Wang, L.-P.; Pande, V. S.; Head-Gordon, T.; Ponder, J. W. Revised Parameters for the AMOEBA Polarizable Atomic Multipole Water Model. J. Phys. Chem. B 2015, 119, 9423-9437.

(7) Levitt, M.; Hirshberg, M.; Sharon, R.; Laidig, K. E.; Daggett, V. Calibration and Testing of a Water Model for Simulation of the Molecular Dynamics of Proteins and Nucleic Acids in Solution. J. Phys. Chem. B 1997, 101, 5051-5061.

(8) Waheed, Q.; Edholm, O. Quantum Corrections to Classical Molecular Dynamics Simulations of Water and Ice. J. Chem. Theory Comput. 2011, 7, 2903-2909. 
(9) Yeh, K.-Y.; Huang, S.-N.; Chen, L.-J.; Lin, S.-T. Diffusive and quantum effects of water properties in different states of matter. J. Chem. Phys. 2014, 141, 044502.

(10) Pascal, T. A.; Schärf, D.; Jung, Y.; Kühne, T. D. On the absolute thermodynamics of water from computer simulations: A comparison of first-principles molecular dynamics, reactive and empirical force fields. J. Chem. Phys. 2012, 13\%, 244507.

(11) Millot, M.; Hamel, S.; Rygg, J. R.; Celliers, P. M.; Collins, G. W.; Coppari, F.; Fratanduono, D. E.; Jeanloz, R.; Swift, D. C.; Eggert, J. H. Experimental evidence for superionic water ice using shock compression. Nature Physics 2018, 14, 297-302.

(12) Naserifar, S.; Goddard, W. A. The quantum mechanics-based polarizable force field for water simulations. J. Chem. Phys. 2018, 149, 174502.

(13) Naserifar, S.; Goddard, W. A. Anomalies in Supercooled Water at 230 K Arise from a 1D Polymer to 2D Network Topological Transformation. J. Phys. Chem. Lett. 2019, 10, 6267-6273.

(14) Naserifar, S.; Oppenheim, J. J.; Yang, H.; Zhou, T.; Zybin, S.; Rizk, M.; Goddard, W. A. Accurate non-bonded potentials based on periodic quantum mechanics calculations for use in molecular simulations of materials and systems. J. Chem. Phys. 2019, 151, 154111.

(15) Kim, Q.; Jhe, W. Interfacial thermodynamics of spherical nanodroplets: molecular understanding of surface tension via a hydrogen bond network. Nanoscale 2020, 12, $18701-18709$.

(16) Caro, M. A.; Lopez-Acevedo, O.; Laurila, T. Redox Potentials from Ab Initio Molecular Dynamics and Explicit Entropy Calculations: Application to Transition Metals in Aqueous Solution. J. Chem. Theory Comput. 2017, 13, 3432-3441. 
(17) Overduin, S. D.; Patey, G. N. Comparison of simulation and experimental results for a model aqueous tert-butanol solution. J. Chem. Phys. 2017, 147, 024503.

(18) Engelmann, S.; Hentschke, R. Specific heat capacity enhancement studied in silica doped potassium nitrate via molecular dynamics simulation. Sci. Rep. 2019, 9, 7606.

(19) Pascal, T. A.; Lin, S.-T.; Goddard III, W. A. Thermodynamics of liquids: standard molar entropies and heat capacities of common solvents from 2PT molecular dynamics. Phys. Chem. Chem. Phys. 2011, 13, 169-181.

(20) Caleman, C.; van Maaren, P. J.; Hong, M.; Hub, J. S.; Costa, L. T.; van der Spoel, D. Force Field Benchmark of Organic Liquids: Density, Enthalpy of Vaporization, Heat Capacities, Surface Tension, Isothermal Compressibility, Volumetric Expansion Coefficient, and Dielectric Constant. J. Chem. Theory Comput. 2012, 8, 61-74.

(21) Greff da Silveira, L.; Jacobs, M.; Prampolini, G.; Livotto, P. R.; Cacelli, I. Development and Validation of Quantum Mechanically Derived Force-Fields: Thermodynamic, Structural, and Vibrational Properties of Aromatic Heterocycles. J. Chem. Theory Comput. 2018, 14, 4884-4900.

(22) Huang, S.-N.; Pascal, T. A.; Goddard, W. A.; Maiti, P. K.; Lin, S.-T. Absolute Entropy and Energy of Carbon Dioxide Using the Two-Phase Thermodynamic Model. J. Chem. Theory Comput. 2011, 7, 1893-1901.

(23) Chen, P.; Nishiyama, Y.; Wohlert, J.; Lu, A.; Mazeau, K.; Ismail, A. E. Translational Entropy and Dispersion Energy Jointly Drive the Adsorption of Urea to Cellulose. J. Phys. Chem. B 2017, 121, 2244-2251.

(24) Chen, M.; Pendrill, R.; Widmalm, G.; Brady, J. W.; Wohlert, J. Molecular Dynamics Simulations of the Ionic Liquid 1-n-Butyl-3-Methylimidazolium Chloride and Its Binary Mixtures with Ethanol. J. Chem. Theory Comput. 2014, 10, 4465-4479. 
(25) Doherty, B.; Zhong, X.; Gathiaka, S.; Li, B.; Acevedo, O. Revisiting OPLS Force Field Parameters for Ionic Liquid Simulations. J. Chem. Theory Comput. 2017, 13, 61316145 .

(26) Doherty, B.; Zhong, X.; Acevedo, O. Virtual Site OPLS Force Field for ImidazoliumBased Ionic Liquids. J. Phys. Chem. B 2018, 122, 2962-2974.

(27) Doherty, B.; Acevedo, O. OPLS Force Field for Choline Chloride-Based Deep Eutectic Solvents. J. Phys. Chem. B 2018, 122, 9982-9993.

(28) Wohlert, J. Vapor Pressures and Heats of Sublimation of Crystalline $\beta$-Cellobiose from Classical Molecular Dynamics Simulations with Quantum Mechanical Corrections. J. Phys. Chem. B 2014, 118, 5365-5373.

(29) Bregado, J. L.; Tavares, F. W.; Secchi, A. R.; Segtovich, I. S. V. Thermophysical Properties of Amorphous-Paracrystalline Celluloses by Molecular Dynamics. Macromol. Theory Simul. 2020, 29, 2000007.

(30) Lai, P.-K.; Hsieh, C.-M.; Lin, S.-T. Rapid determination of entropy and free energy of mixtures from molecular dynamics simulations with the two-phase thermodynamic model. Phys. Chem. Chem. Phys. 2012, 14, 15206-15213.

(31) Pascal, T. A.; Goddard, W. A. Interfacial Thermodynamics of Water and Six Other Liquid Solvents. J. Phys. Chem. B 2014, 118, 5943-5956.

(32) Varanasi, S. R.; Subramanian, Y.; Bhatia, S. K. High Interfacial Barriers at Narrow Carbon Nanotube-Water Interfaces. Langmuir 2018, 34, 8099-8111.

(33) Shrestha, B. R.; Pillai, S.; Santana, A.; Donaldson Jr., S. H.; Pascal, T. A.; Mishra, H. Nuclear Quantum Effects in Hydrophobic Nanoconfinement. J. Phys. Chem. Lett. 2019, $10,5530-5535$. 
(34) Mukherjee, S.; Bagchi, B. Entropic Origin of the Attenuated Width of the Ice-Water Interface. J. Phys. Chem. C 2020, 124, 7334-7340.

(35) Merinov, B. V.; Naserifar, S.; Zybin, S. V.; Morozov, S.; Goddard, W. A.; Lee, J.; Lee, J. H.; Han, H. E.; Choi, Y. C.; Kim, S. H. Li-diffusion at the interface between Li-metal and [Pyr14][TFSI]-ionic liquid: Ab initio molecular dynamics simulations. $J$. Chem. Phys. 2020, 152, 031101.

(36) Rokoni, A.; Sun, Y. Probing the temperature profile across a liquid-vapor interface upon phase change. J. Chem. Phys. 2020, 153, 144706.

(37) Yoon, T. J.; Ha, M. Y.; Lee, W. B.; Lee, Y.-W. "Two-Phase" Thermodynamics of the Frenkel Line. J. Phys. Chem. Lett. 2018, 9, 4550-4554.

(38) Yoon, T. J.; Ha, M. Y.; Lazar, E. A.; Lee, W. B.; Lee, Y.-W. Topological Characterization of Rigid-Nonrigid Transition across the Frenkel Line. J. Phys. Chem. Lett. 2018, 9, 6524-6528.

(39) Yoon, T. J.; Patel, L. A.; Ju, T.; Vigil, M. J.; Findikoglu, A. T.; Currier, R. P.; Maerzke, K. A. Thermodynamics, dynamics, and structure of supercritical water at extreme conditions. Phys. Chem. Chem. Phys. 2020, 22, 16051-16062.

(40) Ghosh, K.; Krishnamurthy, C. V. Frenkel line crossover of confined supercritical fluids. Sci. Rep. 2019, 9, 14872.

(41) Desjarlais, M. P. First-principles calculation of entropy for liquid metals. Phys. Rev. E 2013, 88, 062145 .

(42) Caro, M. A.; Laurila, T.; Lopez-Acevedo, O. Accurate schemes for calculation of thermodynamic properties of liquid mixtures from molecular dynamics simulations. J. Chem. Phys. 2016, 145, 244504. 
(43) Sun, T.; Xian, J.; Zhang, H.; Zhang, Z.; Zhang, Y. Two-phase thermodynamic model for computing entropies of liquids reanalyzed. J. Chem. Phys. 2017, 147, 194505.

(44) Persson, R. A. X.; Pattni, V.; Singh, A.; Kast, S. M.; Heyden, M. Signatures of Solvation Thermodynamics in Spectra of Intermolecular Vibrations. J. Chem. Theory Comput. 2017, 13, 4467-4481.

(45) Pannir Sivajothi, S. S.; Lin, S.-T.; Maiti, P. K. Efficient Computation of Entropy and Other Thermodynamic Properties for Two-Dimensional Systems Using Two-Phase Thermodynamic Model. J. Phys. Chem. B 2019, 123, 180-193.

(46) Dietschreit, J. C. B.; Peters, L. D. M.; Kussmann, J.; Ochsenfeld, C. Identifying Free Energy Hot-Spots in Molecular Transformations. J. Phys. Chem. A 2019, 123, 21632170 .

(47) Li, S.; Pokuri, B. S. S.; Ryno, S. M.; Nkansah, A.; De'Vine, C.; Ganapathysubramanian, B.; Risko, C. Determination of the Free Energies of Mixing of Organic Solutions through a Combined Molecular Dynamics and Bayesian Statistics Approach. J. Chem. Inf. Model 2020, 60, 1424-1431.

(48) Bernhardt, M. P.; Dallavalle, M.; der Vegt, N. F. A. V. Application of the 2PT model to understanding entropy change in molecular coarse-graining. Soft Mater. 2020, o, $1-16$.

(49) Peters, L. D. M.; Dietschreit, J. C. B.; Kussmann, J.; Ochsenfeld, C. Calculating free energies from the vibrational density of states function: Validation and critical assessment. J. Chem. Phys. 2019, 150, 194111.

(50) Jorgensen, W. L. Optimized intermolecular potential functions for liquid alcohols. J. Phys. Chem. 1986, 90, 1276-1284. 
(51) Jorgensen, W. L.; Maxwell, D. S.; Tirado-Rives, J. Development and Testing of the OPLS All-Atom Force Field on Conformational Energetics and Properties of Organic Liquids. J. Am. Chem. Soc. 1996, 118, 11225-11236.

(52) Cadena, C.; Zhao, Q.; Snurr, R. Q.; Maginn, E. J. Molecular Modeling and Experimental Studies of the Thermodynamic and Transport Properties of Pyridinium-Based Ionic Liquids. J. Phys. Chem. B 2006, 110, 2821-2832.

(53) Lagache, M.; Ungerer, P.; Boutin, A.; Fuchs, A. H. Prediction of thermodynamic derivative properties of fluids by Monte Carlo simulation. Phys. Chem. Chem. Phys. 2001, 3, 4333-4339.

(54) Lagache, M.; Ungerer, P.; Boutin, A. Prediction of thermodynamic derivative properties of natural condensate gases at high pressure by Monte Carlo simulation. Fluid Ph. Equilibria 2004, 220, 211 - 223.

(55) Ungerer, P.; Collell, J.; Yiannourakou, M. Molecular Modeling of the Volumetric and Thermodynamic Properties of Kerogen: Influence of Organic Type and Maturity. Energy Fuels 2015, 29, 91-105.

(56) Gong, Z.; Sun, H.; Eichinger, B. E. Temperature Transferability of Force Field Parameters for Dispersion Interactions. J. Chem. Theory Comput. 2018, 14, 3595-3602.

(57) Smith, W. R.; Jirsák, J.; Nezbeda, I.; Qi, W. Molecular simulation of caloric properties of fluids modelled by force fields with intramolecular contributions: Application to heat capacities. J. Chem. Phys. 2017, 147, 034508.

(58) Tenney, C. M.; Massel, M.; Mayes, J. M.; Sen, M.; Brennecke, J. F.; Maginn, E. J. A Computational and Experimental Study of the Heat Transfer Properties of Nine Different Ionic Liquids. J. Chem. Eng. Data 2014, 59, 391-399.

(59) McQuarrie, D.; Simon, J. Molecular Thermodynamics; University Science Books, 1999. 
(60) Berta, D.; Ferenc, D.; Bakó, I.; Madarász, A. Nuclear Quantum Effects from the Analysis of Smoothed Trajectories: Pilot Study for Water. J. Chem. Theory Comput. 2020, $16,3316-3334$.

(61) Bakó, I.; Ádám Madarász,; Pusztai, L. Nuclear quantum effects: Their relevance in neutron diffraction studies of liquid water. J. Mol. Liq. 2021, 325, 115192.

(62) Abraham, M. J.; Murtola, T.; Schulz, R.; Páll, S.; Smith, J. C.; Hess, B.; Lindahl, E. GROMACS: High performance molecular simulations through multi-level parallelism from laptops to supercomputers. SoftwareX 2015, 1-2, 19 - 25.

(63) Engineering ToolBox, Water - Specific Heat. 2004; https:// Www.engineeringtoolbox.com/specific-heat-capacity-water-d_660.html, accessed January 2021.

(64) Engineering ToolBox, Methanol - Specific Heat. 2018; https:// wWw.engineeringtoolbox.com/methanol-CH3OH-specific-heat-capacity-CpCv-isobaric-isochoric-d_2103.html, accessed January 2021.

(65) Engineering ToolBox, Ethanol - Specific Heat. 2018; https:// WwW.engineeringtoolbox.com/specific-heat-capacity-ethanol-Cp-Cvisobaric-isochoric-ethyl-alcohol-d_2030.html, accessed January 2021.

(66) Frenkel, D.; Smit, B. Understanding Molecular Simulation: From Algorithms to Applications, 2nd ed.; Computational Science Series; Academic Press: San Diego, 2002; Vol. 1.

(67) Wang, J.; Hou, T. Application of molecular dynamics simulations in molecular property prediction II: Diffusion coefficient. J. Comput. Chem. 2011, 32, 3505-3519. 


\section{Graphical TOC Entry}

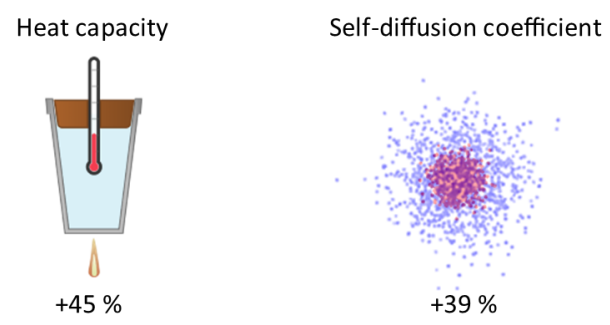

Relative error of typical numerical integration

New estimations for more than 100 organic liquids 